\title{
USO DE REVESTIMENTO ATIVO EM FRUTOS: UMA TECNOLOGIA EMERGENTE
}

\section{USE OF ACTIVE COATING IN FRUITS: AN EMERGING TECHNOLOGY}

\author{
Isamara Reis Gomes ${ }^{1}$; Éder Dutra Resende ${ }^{2}$; Daniele Pereira do Amaral ${ }^{3}$;
}

\begin{abstract}
Resumo
O uso de revestimento ativo para a conservação pós-colheita de frutos é uma tecnologia emergente e coerente com os compromissos da sociedade com a sustentabilidade do planeta, visto que com o grande acumulo de resíduos de embalagens não biodegradáveis, junto com a dificuldade de reciclagem da maior parte dessas, o estímulo de desenvolver embalagens biodegradáveis a partir de fontes renováveis e contribuir para a preservação da qualidade dos frutos é uma alternativa viável e promissora. Entre os polímeros naturais, o amido vem ganhando destaque devido sua grande disponibilidade em escala mundial, alto rendimento de extração, valor nutricional, baixo custo, biodegrabilidade e biocompatilidade. O objetivo deste trabalho foi apresentar esta tecnologia emergente e suas aplicações na conservação de diferentes frutos. $\mathrm{O}$ uso de revestimento ativo em frutas tem se mostrado uma tecnologia na pós - colheita com grande potencial para a conservação do tempo de vida de frutas, visto que garantem um aumento do tempo de vida de prateleira das frutas, são sustentáveis, de fácil aquisição e de custo acessível, porém é necessário maior incentivo em pesquisas com revestimento ativos e desenvolvimento de novos trabalhos para ampliar a aplicação e desenvolvimento de revestimentos ativos.
\end{abstract}

Palavras-Chave: Revestimento ativo, conservação pós- colheita e amido.

\begin{abstract}
The use of active coating for post-harvest fruit conservation is an emerging technology and consistent with society's commitments to the sustainability of the planet, given that with the large accumulation of non-biodegradable packaging waste, along with the difficulty of recycling the most of these, the incentive to develop biodegradable packaging from renewable sources and contribute to the preservation of fruit quality is a viable and promising alternative. Among natural polymers, starch has been gaining prominence due to its great availability on a world scale, high extraction yield, nutritional value, low cost, biodegradability and biocompatibility. The objective of this work was to present this emerging technology and its applications in the conservation of different fruits. The use of active coating on fruits has proven to be a post-harvest technology with great potential for conserving the life of fruits, since they guarantee an increase in the shelf life of fruits, they are sustainable, easy to acquire and of affordable cost, but greater incentive is needed in research with active coatings and development of new works to expand the application and development of active coatings.
\end{abstract}

Keywords: Active coating, post-harvest conservation and starch.

\footnotetext{
${ }^{1}$ Mestranda Produção Vegetal, UENF, isamarareisgomes@gmail.com

2 Professor Dr. na área de tecnologia de alimentos, UENF, eresende@uenf.br

${ }^{3}$ Doutora em Produção Vegetal, UENF, dani.alimento@gmail.com
} 
GOMES, I.R; RESENDE, E.D e AMARAL, D.P.

\section{Introdução}

Segundo a FAO (2019), o Brasil é considerado o terceiro maior produtor de frutas do mundo, graças as suas condições climáticas propícias consegue produzir uma grande variedade de diversas frutas.

A técnica mais comum e usual para conservação de frutas em sua fase pós - colheita é uso de cadeias de frios, com baixas temperaturas e com atmosfera controlada, a fim de conservar por mais tempo a fruta (COSTA e CLEMENTE, 2012).

$\mathrm{O}$ uso de revestimento ativo em frutos tem sido considerado uma tecnologia emergente, que é capaz de manter as condições do fruto por mais tempo, sem geração de resíduos tóxicos e contribuindo para a preservação da textura e do valor nutricional, reduzindo as trocas gasosas superficiais e a perda ou ganho excessivo de água no fruto (TURHAN, 2010).

A motivação para o aumento de interesse e atividade de pesquisa em filmes e revestimentos biodegradáveis está associada à crescente necessidade do consumidor por alimentos saudáveis e estáveis e também à conscientização em relação aos efeitos ambientais nocivos dos resíduos não biodegradáveis resultantes das embalagens (HASSAN et al., 2018).

Segundo Maia (2000), os revestimentos comestíveis são películas que apresentam espessuras variadas, provenientes de substâncias naturais ou sintéticas, que não apresentam riscos à saúde do consumidor, por passarem pelo trato gastrointestinal sem causar nenhum tipo de dano.

O uso de revestimentos comestíveis na conservação de frutos, intactos ou minimamente processados, em pós-colheita, é considerado uma tecnologia emergente e de grande potencial, principalmente para aplicações sobre frutos de origem tropical (ASSIS E BRITO, 2014). Seu uso proporciona mais vantagens quando comparado com os materiais sintéticos, sendo atóxico, podem ser incorporados com aditivos, retardam a perda de água e são de baixo custo. Atualmente, pesquisas têm levado ao desenvolvimento de novas abordagens ambientalmente sustentáveis com base em polímeros biodegradáveis, que não só convertem os subprodutos da indústria de alimentos em valor agregado a componentes formadores de filme, mas também, reduzem os requisitos de embalagem (MAQBOOL ET AL., 2011).

Filmes e revestimentos biodegradáveis vêm sendo bastante utilizados ao longo dos anos, pois apresentam diversos efeitos quando aplicados em frutas e hortaliças, como: retarda a perda de umidade; diminui as trocas gasosas; aumenta a integridade estrutural, promovendo maior proteção física contra injúrias; retém componentes voláteis, constituintes do odor e do 
GOMES, I.R; RESENDE, E.D e AMARAL, D.P.

sabor; e atua como veículo de aditivos alimentícios, como, por exemplo, agentes antimicrobianos e antioxidantes (SALGADO et al., 2015).

\section{Desenvolvimento}

\section{Diversos tipos de revestimento}

As coberturas e revestimentos comestíveis podem ter origem animal ou vegetal, ou formarem um composto com a combinação de ambas. Os mais empregados são polissacarídeos, ceras (lipídios) e proteínas e o tipo a ser utilizado, depende do objetivo desejado com o revestimento aplicado e das características do produto a ser revestido (ASSIS E BRITO, 2014). Os materiais empregados nos revestimentos podem ser classificados em: hidrofóbicos e hidrofílicos (ASSIS ET AL., 2008).

Hidrofílicos: possuem em sua estrutura grupos amino ou hidroxila e carboxila $(\mathrm{OH}$, $\mathrm{COO}-, \mathrm{NH}_{3}$ ) caracterizados por ligações covalentes polares. Possuem cadeia carbônica com sítios parcialmente carregados positivamente e outros carregados negativamente o que favorece o acúmulo e o rearranjo de moléculas polares, e principalmente da água, em torno desses sítios. São representados por polissacarídeos, como a celulose, a quitina, a goma xantana, a goma guar, a pectina, o amido e os polissacarídeos polieletrólitos, como a carboximetilcelulose, a quitosana e o alginato. Já os materiais hidrofóbicos são formados por moléculas cujas ligações tendem a ser eletricamente neutras, que não configuram regiões polares definidas. Na presença de água esses materiais tendem a se aglomerarem e excluir as moléculas polares de sua redondeza. São representados pelas proteínas hidrofóbicas, óleos e ácidos graxos, em que predominam substituintes de cadeia alifática, parafinas e alcoóis de cadeia longa (ASSIS E BRITO, 2014).

As coberturas hidrofílicas são mais indicadas para superfícies fatiadas, frutas com aspectos brilhantes que apresentem alta molhabilidade ou presença de cargas superficiais. Por terem afinidade por água, as coberturas hidrofílicas preservam o aspecto hidratado, mantendo por mais tempo a superfície brilhante (ASSIS E BRITO, 2014).

Segundo dados publicados na literatura diversos são os materiais que podem ser empregados na formação de revestimento, utilizados em diferentes frutos na condição intacta ou fatiada para o prolongamento da vida útil. Foram utilizados para maçã quitosana e alginato (PILON ET AL., 2013; MOLDÃO-MARTINS ET AL., 2003), para o mamão fécula de mandioca e quitosana (CASTRICINI ET AL., 2012; DOTTO ET AL., 2008), para o abacaxi alginato e fécula de mandioca (AZARAKHSH ET AL., 2012; BIERHALS ET AL., 2011), para uva óleo de babosa e getanina com amido (VALVERDE ET AL., 2005; FAKHOURI ET 
GOMES, I.R; RESENDE, E.D e AMARAL, D.P.

AL., 2007), para o morango amido de mandioca (GARCIA ET AL., 2012), para o melão alginato e quitosana (RAYBAUDI-MASSILIA ET AL., 2008; KRASAEKOOPT E MABUMRUNG, 2008), para goiaba quitosana com amido e celulose com carnaúba (SOARES ET AL., 2011; MCGUIRE E HALLMAN 1995), para pêra alginato e amido (OMS-OLIU ET AL., 2008; BOTREL ET AL., 2010), para banana carragina e quitosana (BICO ET AL., 2009; MALMIRI ET AL., 2011), manga fécula de mandioca e quitosana (SCANAVACA JR. ET AL., 2007; CHIEN ET AL., 2007).

O amido pode ser usado em combinação com outros polímeros ou compostos para melhorar as propriedades funcionais da matriz polimérica, que também pode transportar compostos ativos para controlar melhor o prazo de validade do produto (SAPPER E CHIRALT, 2018).

\section{Preparo e aplicação do revestimento ativo}

Segundo Assis e Brito (2014), a formação do revestimento ativo e sua aplicação em frutos podem ser divididos em nove etapas, conforme ilustra a figura 1 , até que se forme uma membrana aderida a cutícula dos frutos ou sobre as estruturas celulósicas das polpas.

O revestimento é obtido pela solubilização dos polímeros em um solvente que geralmente é a água, mas pode ser etanol ou ácido também e pode ou não receber o acréscimo de aditivos, como agentes antimicrobianos, antioxidantes entre outros, obtendo-se uma solução ou dispersão filmogênica (GONTARD et al., 1994).

Durante o processo de formação do revestimento é importante que a evaporação do solvente ocorra de forma espontânea e lenta, para evitar que se formem bolhas e consequentemente uma cobertura porosa, pois isso influenciaria na barreira que o revestimento faz no fruto (STEWARD et al., 2000). 
GOMES, I.R; RESENDE, E.D e AMARAL, D.P.

Figura 1. Etapas para formação do revestimento ativo.

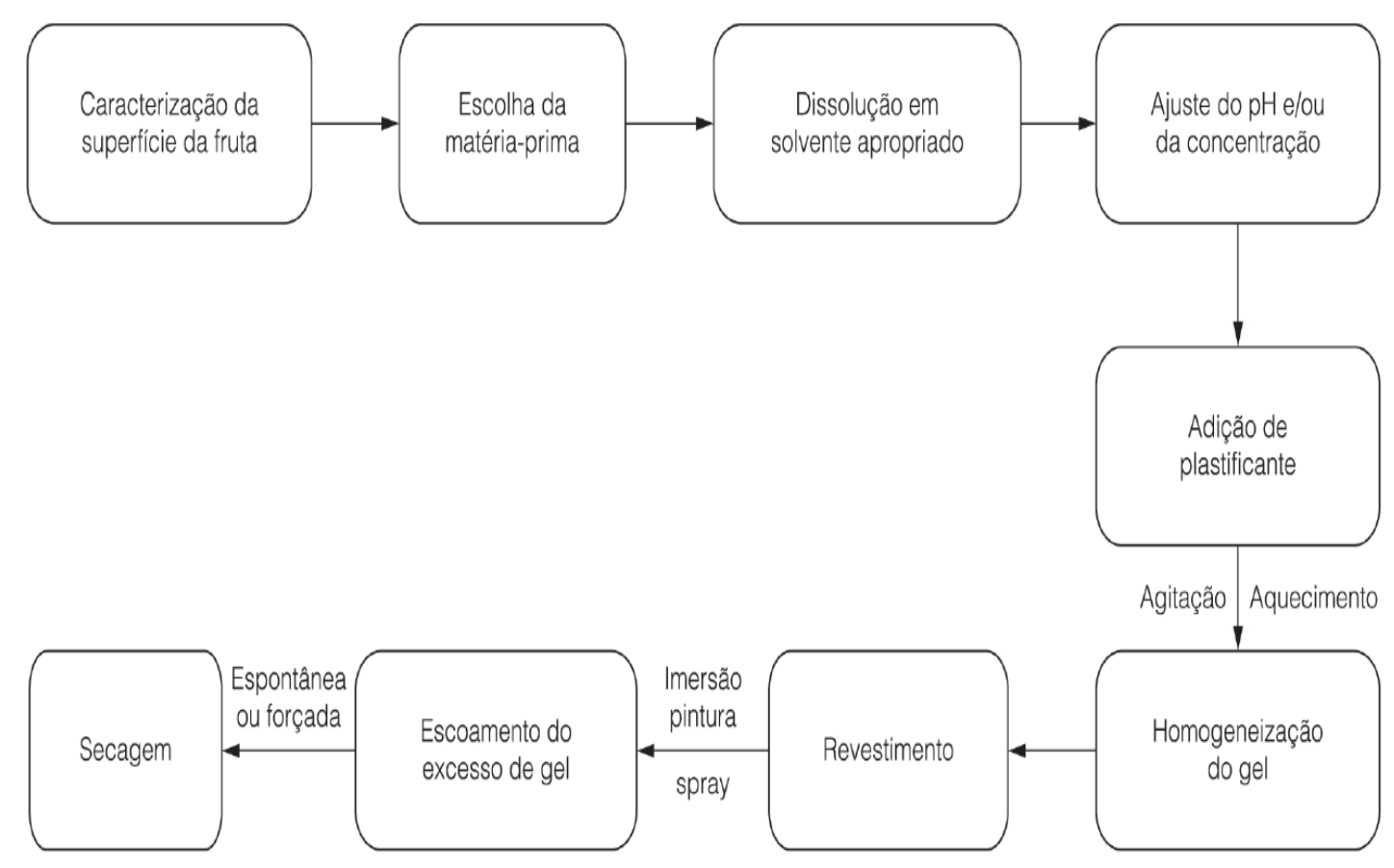

Fonte: Assis e Brito, (2014).

Existem técnicas diferentes para a formação da cobertura em frutos, como uso de pincel, spray e imersão (CHLEBOWSKA SMIGIEL AL., 2007; ANDRADE ET AL., 2012). Mas a técnica mais comum e mais eficiente é o uso da imersão, pois garante que toda a superfície do fruto entre em contato com a solução filmogênica e uma leve agitação nos frutos garante o desprendimento de bolhas e uma deposição de cobertura homogênea.

Ao ser imerso em uma solução filmogênica, o fruto forma uma cobertura pela deposição das espécies poliméricas dissolvidas no meio, estabelecendo ligações, fracas e fortes, com a superfície do fruto. Diversos modelos têm sido propostos para a deposição de estruturas poliméricas e subsequente formação de filmes sobre superfícies sólidas. Nesses modelos, as características do absorvente (a casca) e do absorvato (compostos diluídos na solução filmogênica) são as que definem que tipo de mecanismo será predominante na formação da cobertura (MYERS, 1991; PARIA E KHILAR, 2004). A formação do recobrimento pode ser generalizada segundo a sequência ilustrada na Figura 2. 
GOMES, I.R; RESENDE, E.D e AMARAL, D.P.

Figura 2. Sequência ilustrativa da formação de uma cobertura comestível: (a) imersão do fruto em solução filmogênica (polímeros em solução); (b) atração entre o absorvato (composto diluído na solução filmogênica) e o absorvente (casca); (c) formação da cobertura com evaporação do solvente e reticulação do polímero.

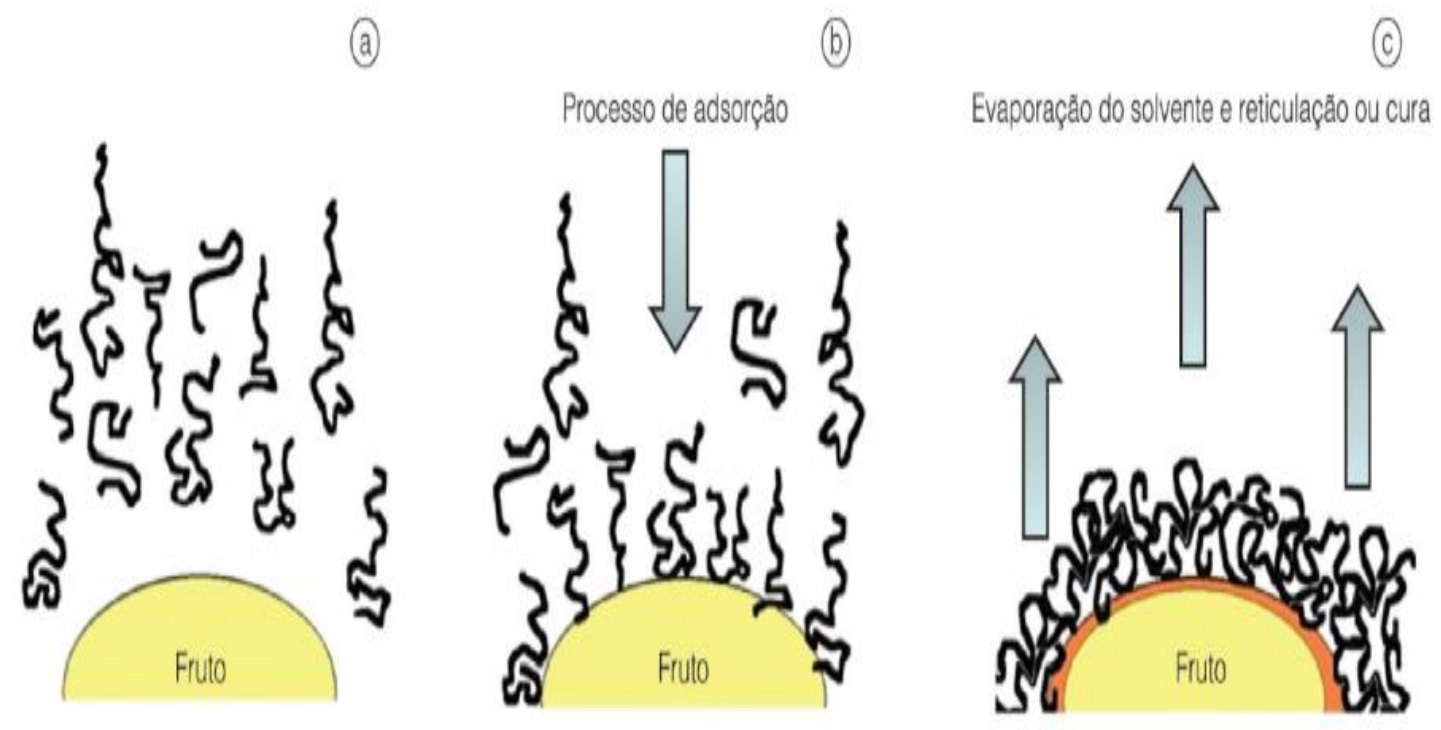

Fonte: Assis e Britto, (2014).

\section{Revestimento a base de amido de mandioca}

O amido é um dos polissacarídeos mais abundantes e importantes na natureza, sendo o mais utilizado para elaboração de coberturas comestíveis em razão do menor custo e alta disponibilidade, fácil manipulação e comestibilidade. Além disso, ele é biodegradável quando lançado no meio ambiente, contribuindo para uma menor poluição ambiental (SAPPER E CHIRALT, 2018).

De modo geral, os amidos contêm cerca de 20 a $30 \%$ de amilose e 70 a $80 \%$ de amilopectina, podendo variar com a fonte botânica, o que confere características específicas ao amido. Variedades mutantes de milho, denominadas de amidos com alto teor de amilose, apresentam um teor de amilose de até $85 \%$, enquanto as variedades comerciais costumam apresentar no máximo 60\%. Em contra partida, certos amidos cerosos contêm apenas amilopectina (CEREDA, 2002). 
GOMES, I.R; RESENDE, E.D e AMARAL, D.P.

Figura 3. Estrutura da amilose.

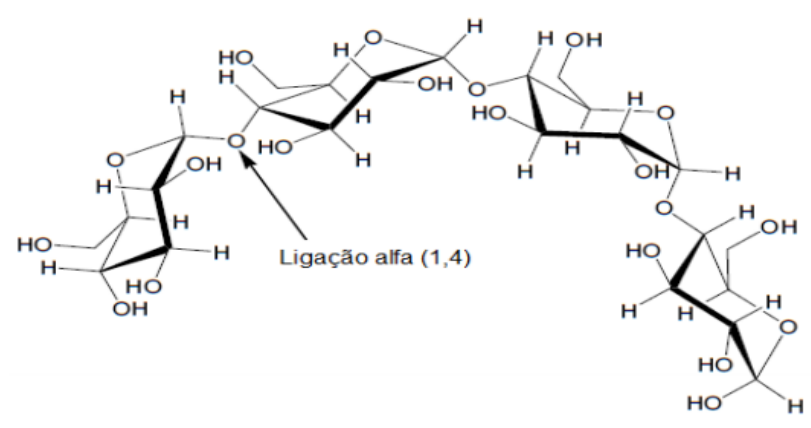

Fonte: Adaptado de WADUGE, 2002.

Figura 4. Estrutura da amilopectina.

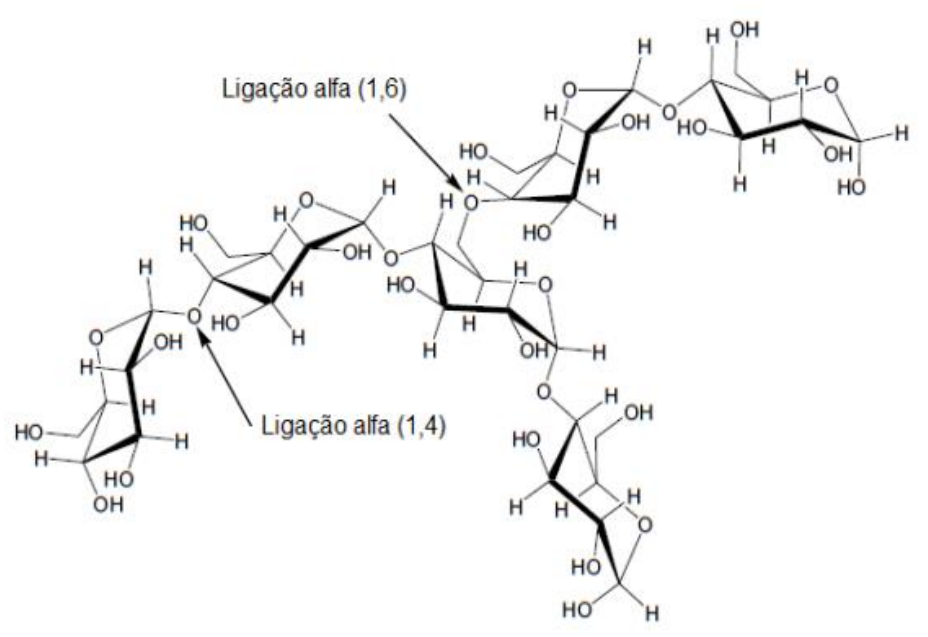

Fonte: Adaptado de WADUGE, 2002.

A fécula (amido) de mandioca é reconhecida por formar películas resistentes e transparentes, sendo eficientes barreiras à perda de água dos alimentos. Na sua forma natural ou modificada, ela é muito utilizada como película visando a conservação após a colheita de produtos frescos (CHITARRA E CHITARRA, 2008).

A formação do revestimento de fécula de mandioca é obtido através da geleificação da fécula durante o aquecimento de uma solução de amido e água à temperatura de $70{ }^{\circ} \mathrm{C}$, promovendo uma ruptura das estruturas cristalinas do grânulo de amido e a absorção de água que entumece a sua estrutura de forma irreversível. Depois da gelatinização do amido e após o resfriamento, quando se atinge a temperatura ambiente, ocorre a retrogradação, ou seja, a 
GOMES, I.R; RESENDE, E.D e AMARAL, D.P.

reorganização das moléculas por ligações de hidrogênio e forma-se um revestimento resistente e transparente (PARKER E RING, 2001).

\section{Discussão}

Segundo Assis e Brito (2008), o uso de revestimentos comestíveis embora esteja em processo de desenvolvimento, tem apresentado resultados significativos, como uma prática auxiliar na conservação de produtos perecíveis e, principalmente, daqueles minimamente processados, cujo tempo de prateleira é consideravelmente reduzido em função dos processos aos quais esses produtos foram submetidos. Os revestimentos comestíveis também são veículo para a incorporação de ingredientes funcionais, tais como antioxidantes, compostos aromáticos, agentes antimicrobianos e nutracêuticos (óleos essenciais) (MORADI ET AL., 2012).

Revestimento à base de fécula de mandioca com concentrações de $2 \%$ e $4 \%$ utilizados em mamão formosa manteve o teor de sólidos solúveis totais, acidez titulável, promoveu o aumento do índice de maturação e de vitamina $\mathrm{C}$, além de reduzir a perda de massa, diâmetro, espessura, firmeza da fruta e polpa (NUNES ET AL., 2017).

Em morangos o revestimento de fécula de mandioca na concentração de $3 \%$ foi eficiente para reduzir a taxa de respiração dos frutos, retardar a perda de peso e firmeza de morangos durante o armazenamento de 12 dias (GARCIA ET AL., 2012).

O revestimento com fécula de mandioca nas concentrações de 3 e $5 \%$ em mamões retardam o desenvolvimento da coloração amarela da casca, porém este desenvolvimento é parcial mantendo os frutos firmes por mais tempo, os frutos apresentam menor perda de massa fresca e reduz a taxa respiratória (CASTRICINI ET AL., 2010).

Segundo Pereira et al. (2006), frutos de mamão formosa tiveram sua vida útil póscolheita prolongada em quatro dias com revestimentos comestíveis à base de fécula de mandioca a $1 \%$ e $3 \%$, sem terem sua qualidade prejudicada em função do retardamento do processo de maturação.

\section{Considerações Finais}

O uso de revestimento ativo em frutas tem se mostrado uma tecnologia emergente na área de pós - colheita e com grande potencial para a conservação do tempo de vida de frutos, visto que garantem um aumento do tempo de vida de prateleira das frutos, são sustentáveis, de fácil aquisição e de custo acessível. 
GOMES, I.R; RESENDE, E.D e AMARAL, D.P.

Dentre os polímeros usados, vale destacar o amido que apresenta bons resultados no prolongamento da vida útil de diferentes frutos, inclusive do mamão. Porém é necessário maior incentivo em pesquisas com revestimento ativo e desenvolvimento de novos trabalhos para ampliar a aplicação e desenvolvimento de revestimentos ativos com novas fontes de polímeros e utilizá-los em novos frutos e em outros alimentos.

Ainda que seja improvável que os filmes e revestimentos ativos possam substituir a embalagem sintética convencional, pesquisas são necessárias e podem ajudar a diminuir os milhões de toneladas de plásticos que são consumidos na atualidade, e minimizar os danos que a embalagem convencional provocam na qualidade de vida no planeta.

\section{Referências}

ANDRADE, R. D.; SKURTYS, O.; OSORIO, F. A. Atomizing spray systems for application of edible coatings. Comprehensive Reviews in Food Science and Food Safety, Chicago, v. 11, n. 3, p. 323-337, 2012.

ASSIS, O. B. G.; FORATO, L. A.; BRITTO, D. Revestimentos comestíveis protetores em frutos minimamente processados. Higiene Alimentar, São Paulo, v. 22, n. 160, p. 99-106, 2008 .

ASSIS, ODILIO BENEDITO GARRIDO; BRITTO, DOUGLAS de. Revisão: coberturas comestíveis protetoras em frutas: fundamentos e aplicações. Brazilian Journal of Food Technology, v. 17, n. 2, p. 87-97, 2014.

AZARAKHSH, N.; OSMAN, A.; GHAZALI, H. M.; TAN, C. P.; MOHD-ADZAHAN, N. Optimization of alginate and gellan based edible coating formulations for fresh-cut pineapples. International Food Research Journal, Selangor, v. 19, n. 1, p. 279-285, 2012.

BICO, S. L. S.; RAPOSO M. F. J.; MORAIS, R. M. S. C.; MORAIS, A. M. M. B. Combined effects of chemical dip and/or carrageenan coating and/or controlled atmosphere on quality of fresh-cut banana. Food Control, Amsterdam, v. 20, n. 5, p. 508-514, 2009. 
GOMES, I.R; RESENDE, E.D e AMARAL, D.P.

BIERHALS, V. S.; CHIUMARELLI, M.; HUBINGER, M. D. Effect of cassava starch coating on quality and shelf life of fresh-cut pineapple (Ananas comosus L. Merril cv “Pérola”). Journal of Food Science, Chicago, v. 76, n. 1, p. E62-E72, 2011.

BOTREL, D. A.; SOARES, N. F. F.; CAMilloto, G. P.; FERNANDES. R. V. B. Revestimento ativo de amido na conservação pós-colheita de pera Williams minimamente processada. Ciência Rural, Santa Maria, v. 40, n. 8, p.1814-1820, 2010.

CARNEIRO, Lucia Cesar. Revestimentos à base de amido na conservação pós-colheita de pedúnculos de caju anão precoce e goiabas paluma. Orientadora: $\operatorname{Prof}^{a} \operatorname{Dr}^{\mathrm{a}}$ Angelita da Silveira Moreira. Tese de doutorado (Programa de Pós graduação em Ciência e tecnologia de alimentos) - Faculdade de agronomia Eliseu Maciel, universidade Federal de Pelotas, Pelotas, 2019.

CASTRICINI, A.; CONEGLIAN, R. C. C.; DELIZA. R. Starch edible coating of papaya: effect on sensory characteristics. Ciência e Tecnologia dos Alimentos, Campinas, v. 32, n. 1, p. 84-92, 2012.

CASTRICINI, ARIANE; CONEGLIAN, REGINA CELI CAVESTRÉ; DA SILVA VASCONCELLOS, MARCO ANTONIO. Qualidade e amadurecimento de mamões 'golden'revestidos por película de fécula de mandioca. Revista Trópica: Ciências Agrárias e Biológicas, v. 4, n. 1, 2010.

CEREDA, M. P. Propriedades gerais de amido. São Paulo. Fundação Cargill, v. 1. 221p. (Série: Culturas de tuberosas amiláceas latino-americanas), 2002.

CHIEN, P-J.; SHEU, F.; YANG, F-H. Effects of edible chitosan coating on quality and shelf life of sliced mango fruit. Journal of Food Engineering, Amsterdam, v. 78, n. 1, p. 225-229, 2007.

CHITARRA, M. I. F.; CHITARRA, A. B. Pós-colheita de frutas e hortaliças: fisiologia e manuseio. Lavras, Editora UFLA, 2008. 
GOMES, I.R; RESENDE, E.D e AMARAL, D.P.

CHLEBOWSKA - SMIGIEL, A.; GNIEWOSZ, M.; SWINCZAK, E. An attempt to apply a pullulan and pullulan-protein coatings to prolong apples shelf-life stability. Acta Scientiarum Polonorum Technologia Alimentaria, Poznan, v. 6, n. 1, p. 49-56, 2007.

COSTA, J. M. C. DA; CLEMENTE, E. Refrigeration and cold chain effect on fruit shelf life. In: RODRIGUES, S.; FERNANDES, F. A. N. (Ed.). Advances in fruit processing technologies. Boca Taton: CRC Press, p. 287-330. http://dx.doi.org/10.1201/ b12088-13, 2012.

DOTTO, G. L.; GREVINELI, A. C.; OlIVEIRA, A.; PONS, G.; PINTO, L. A. A. Uso de quitosana como fi lme microbiológico para o aumento da vida útil de mamões papaia. In: Congresso de iniciação científica e encontro de pós-graduação, 17., 2008, Pelotas. Anais eletrônicos. Pelotas: Universidade Federal de Pelotas, 2008. Disponível em <www.ufpel.edu.br/ cic/2008/cd/pages/pdf/CA. $>$ Acessado em: maio de 2020.

FAKHOURI, F. M., FONTES, L. C. B., GONÇALVES, P. V. D. M., MILANEZ, C. R., STEEL, C. J., \& COLLARES-QUEIROZ, F. P. Filmes e coberturas comestíveis compostas à base de amidos nativos e gelatina na conservação e aceitação sensorial de uvas Crimson. Food Science and Technology, 27(2), 369-375, 2007.

FAO. Food and Agriculture Organization of the United Nations, 2019. Disponível em: < http://www.fao.org/statistics/en/>. Acessado em: Maio de 2020.

GARCIA, L. C.; PEREIRA, L. M.; SARANTÓPOUlOS, C. I. G. L.; HUBINGER M. D. Effect of antimicrobial starch edible coating on shelf-life of fresh strawberries. Packaging Technology and Science, Hoboken, v. 25, n. 7, p. 413-425, 2012.

GONTARD, N.; DUCHEZ, C.; CUQ, J.; GUILBERT, S. Edible composite films of wheat gluten and lipids: Water vapor permeability and other physical properties. International Journal Food Science Technology, v. 29, n. 39-50, 1994.

HASSAN, B.; CHATHA, S. A. S.; HUSSAIN, A. I.; ZIA, K. M.; \& AKHTAR, N. Recent advances on polysaccharides, lipids and protein based edible films and coatings: A review. International journal of biological macromolecules, v. 109, p. 1095-1107, HONG, 2018. 
GOMES, I.R; RESENDE, E.D e AMARAL, D.P.

KRASAEKOOPT, W.; MABUMRUNG, J. Microbiological evaluation of edible coated freshcut cantaloupe. Kasetsart Journal (Natural Science), Bangkok, v. 42, n. 3, p. 552-557, 2008 .

MAIA, L. H.; PORTE, A.; SOUZA, V. F. Filmes comestíveis: aspectos gerais, propriedades de barreira a umidade e oxigênio. Boletim do Centro de Pesquisa de Processamento de Alimentos, Curitiba, v. 18, n. 1, p.105-128, 2000.

MALMIRI, H. J.; OSMAN, A.; TAN, C. P.; RAHMAN, R. A. Development of an edible coating based on chitosan-glycerol to delay 'Berangan' banana (Musa sapientum cv. Berangan) ripening process. International Food Research Journal, Selangor, v. 18, n. 3, p. 989-997, 2011.

MAQBOOL, M.; ALI, A.; ALDERSON, P. G.; ZAHID, N.; SIDDIQUI, Y. Postharvest Biology and Technology Postharvest application of gum arabic and essential oils for controlling anthracnose and quality of banana and papaya during cold storage. Postharvest Biology and Technology, v. 62, n. 1, p. 71-76, 2011.

MCGUIRE, R. G.; HALLMAN, G. J. Coating guavas with cellulose or carnauba-based emulsions interferes with postharvest ripening. HortScience, Alexandria, v. 30, n. 2, p. 294$295,1995$.

MOLDÃO-MARTINS, M.; BEIRÃO-DA-COSTA, S. M.; BEIRÃO DA - COSTA, M. L. The effects of edible coatings on postharvest quality of the "Bravo de Esmolfe" apple. European Food Research and Technology, Berlin, v. 217, n. 4, p. 325-328, 2003.

MORADI, M., TAJIK, H., ROHANI, S. M. R., OROMIEHIE, A. R., MALEKINEJAD, H., ALIAKBARLU, J., \& HADIAN, M. Characterization of antioxidant chitosan film incorporated with Zataria multiflora Boiss essential oil and grape seed extract. $L W T$-Food Science and Technology, 46(2), 477-484, 2012.

MYERS, D. Surface, interfaces, and colloids: principles and applications. New York: VCH Publishers, 1991 
GOMES, I.R; RESENDE, E.D e AMARAL, D.P.

NUNES, A. C. D., NETO, A. F., NASCIMENTO, I. K., DE OLIVEIRA, F. J., \& MESQUITA, R. V. C. Armazenamento de mamão formosa revestido à base de fécula de mandioca. Revista de Ciências Agrárias, 40(1), 254-263, 2017.

OMS-OLIU, G.; SOLIVA-FORTUNY, R.; MARTÍN-BELLOSO, O. Edible coatings with antibrowning agents to maintain sensory quality and antioxidant properties of fresh-cut pears. Postharvest Biology and Technology, Amsterdam, v. 50, n. 1, p. 87-94, 2008.

PARIA, S.; KHILAR, K. C. A review on experimental studies of surfactant adsorption at the hydrophilic solid-water interface. Advances in Colloid and Interface Science, Amsterdam, v. 110, n. 3, p. 75-95, 2004.

PARKER, R.; RING, S. G., 2001. Aspects of the physical chemistry of starch. Journal of Cereal Science, Norwich, v. 34, p. 1-17, 2001.

PEREIRA, M. E. C., SILVA, A. S. D., BISPO, A. S. D. R., SANTOS, D. B. D., SANTOS, S. B. D., \& SANTOS, V. J. D. Amadurecimento de mamão formosa com revestimento comestível à base de fécula de mandioca. Ciência e Agrotecnologia, 30(6), 1116-1119, 2006.

PILON, L.; SPRICIGO, P. C.; BRITTO, D.; ASSIS, O. B. G.; CALBO, A. G., FERRAUDO, A. S., FERREIRA, M. D. Effects of antibrowning solution and chitosan-based edible coating on the quality of fresh-cut apple. International Journal of Postharvest Technology and Innovation, Geneve, v. 3, n. 2, p. 151-164, 2013.

RAYBAUDI - MASSILIA, R. M.; MOSQUEDA - MELGAR, J.; MARTÍN - BELLOSO, O. Edible alginate-based coating as carrier of antimicrobials to improve shelf-life and safety of fresh-cut melon. International Journal of Food Microbiology, Amsterdam, v. 121, n. 3, p. 313-32, 2008.

SALGADO, P. R.; ORTIZ, C. M.; MUSSO, Y. S.; DI GIORGIO, L.; MAURI, A. N. Edible films and coatings containing bioactives. Current Opinion in Food Science, v. 5, p. 86-92, 2015. 
GOMES, I.R; RESENDE, E.D e AMARAL, D.P.

SAPPER, MAYRA; CHIRALT, AMPARO. Starch-based coatings for preservation of fruits and vegetables. Coatings, v. 8, n. 5, p. 152, 2018.

SCANAVACA JR., L.; FONSECA, N.; PEREIRA, M. E. C. Uso de fécula de mandioca na pós-colheita de manga 'surpresa'. Revista Brasileira de Fruticultura, Jaboticabal, v. 29, n. 1, p. 67-71, 2007.

SOARES, N. F. F.; SILVA, D. F. P.; CAMILlOTO, G. P.; OLIVEIRA, C. P.; PINHEIRO, N. M.; MEDEIROS, E. A. A. Antimicrobial edible coating in post-harvest conservation of guava. Revista Brasileira de Fruticultura, Jaboticabal, v. 33, n. 1, p. 281-289, 2011.

STEWARD, P. A.; HEARN, J.; WILKINSON, M. C. An overview of polymer latex fi lm formation and properties. Advances in Colloid and Interface Science, Amsterdam, v. 86, n. 3, p. 195-267, 2000.

TURHAN, K. N. Is edible coating an alternative to MAP for fresh and minimally processed fruits? Acta Horticulturae, Leuven, v. 876, n. 1, p. 299-305, 2010.

VALVERDE, J. M.; VALERO, D.; MARTÍNEZ-ROMERO, D.; GUILLÉN, F.; CASTILLO, S.; SERRANO, M. Novel edible coating based on aloe vera gel to maintain table grape quality and safety. Journal of Agricultural and Food Chemistry, Easton, v. 53, n. 20, p. 7807-7813. 2005. 